\title{
Beyond Microblogging: Conversation and Collaboration via Twitter
}

\author{
Courtenay Honeycutt \\ Indiana University Bloomington \\ clhoneyc@indiana.edu
}

\author{
Susan C. Herring \\ Indiana University Bloomington \\ herring@indiana.edu
}

\begin{abstract}
The microblogging service Twitter is in the process of being appropriated for conversational interaction and is starting to be used for collaboration, as well. In order to determine how well Twitter supports user-touser exchanges, what people are using Twitter for, and what usage or design modifications would make it (more) usable as a tool for collaboration, this study analyzes a corpus of naturally-occurring public Twitter messages (tweets), focusing on the functions and uses of the@sign and the coherence of exchanges. The findings reveal a surprising degree of conversationality, facilitated especially by the use of @ as a marker of addressivity, and shed light on the limitations of Twitter's current design for collaborative use.
\end{abstract}

\section{Introduction}

Twitter-a web-based microblogging service that allows registered users to send short status update messages to others - is a new social software phenomenon that is attracting attention from the popular press [2, 12] and, increasingly, from scholars [8,9]. Launched in the fall of 2006, Twitter has grown rapidly in popularity in recent months. Compete.com reports that from February to April 2008, U.S. traffic to the site nearly doubled to approximately 1.2 million people per month [3]. Twitter is also popular in other parts of the world, including Japan, Europe, and South America [17].

The stated purpose of Twitter is for users to answer the question: "What are you doing?" [15]. However, in Mischaud's [9] study of 5,767 Twitter messages, $58.5 \%$ of the messages did not address this question. In an alternative appropriation, a growing number of people are using Twitter to interact with others, sometimes in extended exchanges - even though the site was not designed primarily for such use. To facilitate their exchanges, Twitter users have innovated a novel use of the familiar "@” sign as a marker of addressivity [16], as in @courosa to indicate that a message (or "tweet") is addressed to the user ID courosa.

One potentially important role for person-to-person interaction via Twitter is collaboration. Twitter already is being used to disseminate information in institutional settings and to connect groups of people in critical situations [17]. Given its flexibility of access and lightweight architecture, Twitter also has the potential to be used for sharing ideas and coordinating activities, similar to instant messaging [13], yet more dynamic. There is some evidence that this potential is already being realized. Odden [10], in an informal blog poll, found that $2 \%$ of respondents reported using Twitter for "group and project communication." However, Odden's data rely on a convenience sample, visitors to his blog. More in-depth study is needed to assess the collaboration occurring via Twitter. Such research should focus on the ease and sustainability of user-touser exchanges, since the ability to converse is a basic requirement for collaboration. Thus we ask: How well does Twitter support user-to-user exchanges, what are people using Twitter for, and what usage or design modifications would be required to make it (more) usable as a tool for collaboration?

We address these questions by analyzing conversational exchanges on the Twitter public timeline, focusing on the functions and uses of the @ sign. The findings reveal that despite a "noisy" environment and an interface that is not especially conducive to conversational use, short, dyadic exchanges occur relatively often, along with some longer conversations with multiple participants that are surprisingly coherent. These conversations are facilitated in large measure by use of the @ sign as a marker of addressivity (i.e., to direct a tweet to a specific user) and the ability to "follow" other users, which aid users in tracking conversations. In light of this evidence, we consider the suitability of microblogging tools for collaboration and advance recommendations for enhancing their design to better promote conversation and collaboration.

\section{Background}

\subsection{Twitter}

Twitter was created by a San Francisco-based 10person start-up called Obvious and launched in October 2006. Users send messages (called "tweets")limited to 140 characters - to a web interface, where 
they are displayed. Users can indicate whether they wish their tweets to be public-meaning that the messages appear in reverse chronological order on the "public timeline" on Twitter.com's home page and on the individual user's Twitter page (the user's “microblog"), or private - meaning that only those who have subscribed to the user's feed ("followers") are able to see the messages. Tweets can be posted via Twitter.com, text messaging, instant messaging, or from third party clients; the ability to post from mobile phones makes Twitter a mobile application. In addition, Twitter's designers opted to open the service's infrastructure to outside developers, and as of June 2007, over 100 third-party clients had been created [2]. These include Twitteriffic and Twitteroo, which allow users to send and receive tweets via desktop applications.

Twittering appears to be primarily a weekday activity, with the service receiving "more than twice the attention [on a weekday] as [on] a weekend day" [3]. According to Compete [3], as of April 2008, U.S. users tend to be young and male. Twitter attracts users aged 18-24 at nearly twice the rate of an average website; however, this age group appears to be "exploratory" users of the site, visiting only a few times a month. Heavier Twitter users (defined as persons using the site six or more times per month) are between the ages of 25 and 44, a group that Freiert [3] suggests may have "found more value in Twitter and started to ramp up usage."

In perhaps the first study of Twitter use, Java et al. [8] identified three main categories of Twitter users: information sources, friends, and information seekers. Information sources post news and tend to have a large base of "followers"; these sources may be individuals or automated services. Friends is a broad category that encompasses most users, including family, co-workers, and strangers. Finally, information seekers tend to be users who may post rarely but who follow others regularly.

Java et al. also identified several categories of intention to use Twitter, including daily chatter, where users discuss events in their lives or their current thoughts; sharing information or URLs; and reporting news, which includes commenting on current events or automated news agents posting weather or news stories. According to the researchers [8], this latter development "has evolved due to easy access to the developer API [application programming interface]" (p. 8).

The fourth - and most relevant to the present study - category of user intention is conversation. Taking the appearance of the @ sign as an indicator, [8] found that $21 \%$ of the users in their study used Twitter for this purpose, and that one-eighth of the messages $(12.5 \%)$ were part of conversations. Similarly, Mis-
Mischaud [9] found that in his sample, "many postings often read like fragments of virtual conversation" ( $p$. 30). He added that when the @ sign appeared in a tweet, it "was clear that a fellow Twitter user was being interacted with" (p. 30). However, [9] studied a relatively small sample of users $(\mathrm{N}=60)$.

Moreover, it is not self-evident that all uses of the (a) sign in tweets are intended to create conversation, or that all Twitter conversations use the @ sign. Additional factors may need to be accounted for to determine if, and if so, to what extent, twittering is conversational, including the user's intention behind displaying an@ sign and whether@ signs that direct messages to others receive any response.

\subsection{Addressivity}

The innovative use by Twitter early adopters of the (a) sign to direct messages to other users is a form of addressivity, as described by Werry [16] for Internet Relay Chat (IRC). Werry defined addressivity as a user indicating an intended addressee by typing the person's name at the beginning of an utterance, often followed by a colon. He noted that a high degree of addressivity is required in multi-participant public environments such as IRC, because the addressee's attention must be recaptured with every new utterance. Bays [1] observed that addressivity functions in a similar manner in IRC as gaze does in face-to-face conversations, directing the next turn either to one particular person or to the group as a whole. Explicitly addressed turns may be followed by responses directed back to the turn initiator or to the group at large, if the respondent wishes to open the discussion, and thus perform the important work of turn allocation $[11,14]$.

\subsection{Coherence}

Addressivity is a strategy for creating cross-turn coherence online $[5,11]$. Coherence in the context of computer-mediated communication can be defined as sustained, topic-focused, person-to-person exchanges [18].

Coherence is often problematic in CMC, especially in multi-participant, public environments such as chatrooms and discussion forums, in that messages are posted in the order they are received by the system, without regard for what a message is responding to. As a consequence, messages that logically respond to one another are often disrupted by intervening messages [5]. This is in contrast to face-to-face conversations, which tend to have higher degrees of turn adjacencyresponses occur temporally adjacent to the initiating messages [14]. The more participants there are in a conversation, the greater the likelihood that turns will 
be disrupted, and the greater the number of intervening messages between turns. However, the relative incoherence of multi-participant CMC does not make it less popular; on the contrary, the ability to engage in multiple interleaved conversations appears to be appreciated by many CMC users, who may find the level of activity stimulating and the need to match responses to their initiating messages an enjoyable challenge [5].

Twitter is a "noisy" environment, due to the large number of tweets and the speed with which they are posted. This, combined with the fact that tweets are posted in the order received by the system, leads to a high degree of disrupted turn adjacency when a tweet responds to another tweet-much higher than in a typical chatroom or discussion forum. In this noisy environment, use of the@ sign is a useful strategy for relating one tweet to another and, indeed, for making coherent exchanges possible.

\section{Research Questions}

This study poses and addresses five research questions related to the broad question of how much and how effectively Twitter is used for conversation. First, because users from different cultures may display different patterns, we ask:

RQ1: What is the breakdown of the language of tweets across time periods, and to what extent is the @ sign used in tweets in different languages?

Next, to test the assumption in previous Twitter research [8,9] that the presence of the @ sign in an English tweet signifies that it is part of a conversation (i.e., addressed to someone), and to determine if tweets with andwithout@ signs function differently,we ask:

RQ2: How do instances of the@ sign function in English tweets?

RQ3: What do people twitter about, and does it vary for tweets with and without@ signs?

Another indicator of conversationality is the extent to which initiations are followed by responses. Thus we ask:

RQ4: To what extent do English@ messages that are directed to others receive responses, either with orwithout@signs?

Finally, to examine the coherence of extended Twitter exchanges and the role played by@ signs in exchanges, we ask:

RQ5: How long, and how coherent, are interactive exchanges, and to what extent do they make use of the@ sign?

\section{Methodology}

\subsection{Data}

To address these questions, tweets were collected from Twitter.com's public timeline (a feed available to all Twitter users) in four one-hour samples gathered at four-hour intervals, starting at 6 a.m. and ending at 6 p.m. Eastern Standard Time, on January 11, 2008. After determining that it was impossible to refresh the public timeline fast enough or often enough to capture all posted tweets, and after evaluating several thirdparty Twitter clients, we ultimately used a program developed for this study called Twitter Scraper, ${ }^{1}$ which captures tweets in three-second intervals and saves them in a database.

Our goal was to collect all tweets posted to the public timeline during the four hours sampled. However, we were not able to capture them all, for two reasons. First, Twitter Scraper was only able to collect up to 20 tweets per iteration; if more than 20 messages were sent to the server in three seconds, only the first 20 were collected. Second, during periods of heavy activity, Twitter Scraper sometimes took longer than three seconds to gather and return the newest tweets, or the program returned an error message. It is unknown how many individual tweets were missed due to these constraints. Moreover, tweets not included on the public timeline were not captured by Twitter Scraper, so private messages are not part of the sample.

Table 1 shows the number of tweets collected per time period and the number of times Twitter Scraper returned a warning that not all tweets had been captured.

Table 1. Number of tweets captured per time period (Eastern Standard Time)

\begin{tabular}{|l|c|c|c|c|}
\hline Time & \# Tweets & $\begin{array}{c}\text { \# Requests } \\
\text { to Server }\end{array}$ & \# Warnings & $\begin{array}{c}\% \text { Times } \\
\text { Tweets } \\
\text { Missed }\end{array}$ \\
\hline 6 AM & 7,754 & 733 & 41 & 5.59 \\
10 AM & 12,234 & 774 & 219 & 28.29 \\
2 PM & 8,471 & 663 & 108 & 16.29 \\
6 PM & 8,528 & 762 & 67 & 8.79 \\
\hline Total & 36,987 & 2,932 & 435 & -- \\
\hline
\end{tabular}

\footnotetext{
1 Twitter Scraper was developed by Daniel Kutz at Indiana University Bloomington. The tool is available for public use (http://bitlib.org/twitter/index.php); however, it behaves differently now from when we used it to collect data for this study. When the wait between sampling is less than 30 seconds, a result is often returned that there have been no new updates; this did not happen previously. We suspect that it is due to changes to the Twitter architecture, rather than to a decrease in Twitter use.
} 


\subsection{Analytical methods}

To address the first research question, we coded a random sample of 50 tweets from each of the four time periods, for a total of 200 tweets, for a) the language used in the tweet and b) the self-reported geographical location of the sender, which was present in most of the user profiles. Only the language results are presented in this paper. The remaining questions were answered based on the English tweets, which comprise the majority of the sample. Specifically, we focused on English tweets from the 6 p.m. sample, the time period with the most English tweets and that also had relatively few warnings of missed tweets.

For the second research question, content analysis methods were used to code the functions of the @ sign. A grounded theory approach was followed, allowing the categories relevant to @ use to emerge from the data. The following categories were used to categorize the functions of the @ sign:

1) Addressivity: Directs a message to another person

2) Reference: Makes reference to another person, but does not direct a message to him or her. E.g., sooooooooooooo jealous of @strebel and his nap...

3) Emoticon: Used as part of an emoticon. E.g., @_@

4) Email: Used as part of an email address. E.g., ping me at taidlin@microsoft.com

5) Locational 'at': Signals where an entity is located. E.g., Relaxing@ Franks Pizza with the girls.

6) Non-locational 'at': Used to represent the preposition 'at' other than in the sense of location. E.g., 2 energy shots, $i$ want to lift weights, have 3 conversations and@the same time listen to my ipod while doing email

7) Other: Uses not fitting into any other category, including in representations of swear words and metalinguistic references to use of the @ sign on Twitter. E.g., The @ $\# \$ \% \wedge \&$ meeting ended badly.

A single tweet could include multiple@ signs with different functions; in that case, each function of @ was coded separately.

Content analysis was also employed to classify the main content theme of each tweet (RQ3), again using a grounded theory approach to identify the coding categories most characteristic of the data. The following categories were identified and coded; each tweet was coded only once:

1) About addressee: solicits or comments on information relating to the addressee

2) Announce/advertise: announces information to the general readership of Twitter
3) Exhort: directs or encourages other(s) to do something

4) Information for others: posts information apparently intended for others; may be solicited or volunteered

5) Information for self: posts information apparently intended for sender's own use

6) Metacommentary: comments on Twitter or twittering

7) Media use: reports or reflects on media use, especially music

8) Opinion: asserts a subjective or evaluative position

9) Other's experience: solicits, reports on, or comments on information relating to the experience of a third person or persons

10) Self experience: reports or comments on sender's own experience

11) Solicit information: requests information (other than about addressee)

12) Other: miscellaneous other themes, e.g., greetings, nonsense

Interaction analysis methods were used to answer the last two research questions, which involved response rate (RQ4) and the characteristics of extended exchanges (RQ5). We examined English messages (with and without @ signs) that were posted to Twitter within the first 30 minutes of the 6 p.m. sample, and identified all public responses (using the @ sign) that appeared at any time within the hour after the initiating message. All exchanges - defined as a minimum of an initiation and one response-were also identified, and descriptive statistics were calculated regarding their length, timing, and number of participants.

Finally, we used the Dynamic Topic Analysis method [6] to analyze the coherence of longer exchanges. Dynamic topic analysis (DTA) is a technique for analyzing coherence in text-based computermediated discourse. The DTA analyst manually codes the relationship between each proposition and the previous proposition to which it relates - as either narrowly on-topic (T), a parallel shift (P), or a break (B) as well as the semantic distance between them, on a scale of 0 to 4 . The method enables quantification and generates a visualization of the resulting patterns as conversational topics unfold dynamically over time. We used VisualDTA, a Java-based application developed by Herring and Kurtz [7], to automate the generation of visualizations from DTA coding.

DTA was recently employed by Zelenkauskaite and Herring [18] to visualize interactive exchanges via mobile phone text (SMS) messages posted to an Italian interactive television (iTV) program, another very noisy and disruptive communicative environment. 


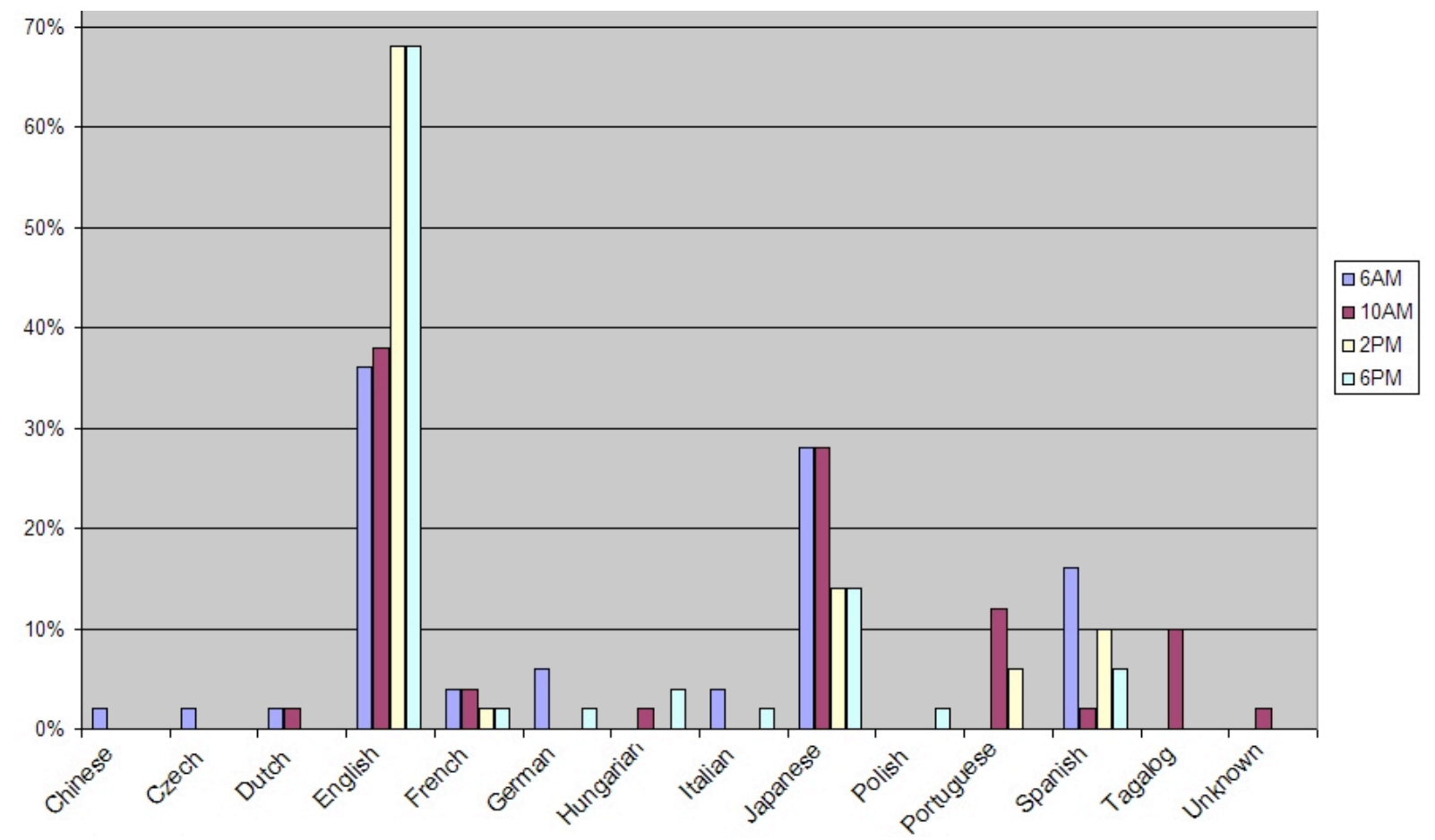

Figure 1. Tweets posted by language and time (all times Eastern Standard Time)

The researchers found that for any given time period, the communication appeared highly incoherent; almost no conversational exchanges seemed to be taking place. When messages unrelated to exchanges between dyads were cleaned from the data, however, extended dyadic conversations could be identified that were moderately coherent. However, they were far less coherent than IM or IRC exchanges visualized using the same method. The present study partially replicates Zelenkauskaite and Herring's [18] methods, so as to be able to compare the Twitter coherence results with those for other CMC conversation.

\section{Findings}

\subsection{Language of tweets}

As might be expected, Twitter posting activity varies according to time zone. English tweets are most common overall, followed by Japanese and Spanish. These results are displayed in Figure 1.

The different language groups appear to make use of the @ sign with equal frequency. Overall, 30\% of tweets in the 6 a.m. and 6 p.m. samples, $31 \%$ of tweets in the 10 a.m. sample, and $32 \%$ of the tweets in the 2 p.m. sample use the @ sign. This is more than twice as high as the rate reported in Java et al. [8], who found that $12.5 \%$ of all tweets in their collection contained an (a) sign. This suggests that interactive uses of Twitter are increasing rapidly and that this is a global trend.

Although English tweets were the most common in all four time periods, they were most frequent $(68 \%)$ in two time periods: 2 p.m. and 6 p.m. For the remaining research questions, we focused on tweets posted in the 6 p.m. sample, as it had fewer warnings of missed tweets from Twitter Scraper than the 2 p.m. sample.

\subsection{Functions of @}

The second research question sought to categorize and quantify the varying uses of the @ sign in English tweets. In the 6 p.m. sample, 1472 English tweets employing an@ sign were found. The breakdown of their functions is summarized in table 2 .

Nearly $91 \%$ of the @ signs appearing in the 6 p.m. sample were used to direct a tweet to a specific addressee. The next most common use of the @ sign was to refer to another user, followed by indicating one's current location. In this sample, 28 users included multiple@ signs in their tweets for different purposes. An analysis of a random sample of 50 English tweets employing the@ sign from the other time periods suggests that the percentages in Table 2 are representative of the larger corpus. 
Table 2. Functions of the @ sign in English tweets

\begin{tabular}{|l|c|c|}
\hline & Number & $\%$ \\
\hline Addressivity & 1339 & $90.96 \%$ \\
\hline Reference & 80 & $5.43 \%$ \\
\hline Locational 'at' & 11 & $0.75 \%$ \\
\hline Non-locational 'at' & 5 & $0.34 \%$ \\
\hline Email & 1 & $0.07 \%$ \\
\hline Emoticon & 1 & $0.07 \%$ \\
\hline Other & 7 & $0.48 \%$ \\
\hline Address / Refer & 20 & $1.36 \%$ \\
\hline Address / Location & 3 & $0.20 \%$ \\
\hline Address / Email & 2 & $0.14 \%$ \\
\hline Address / Other & 1 & $0.07 \%$ \\
\hline Refer / Other & 2 & $0.14 \%$ \\
\hline Total & 1472 & $100.00 \%$ \\
\hline
\end{tabular}

\subsection{Content of tweets with and without @}

We next asked what people twitter about, and if the content of tweets differs when the@ sign is used to direct a message to a specific individual, as opposed to posting it for oneself or the general Twitter readership. The main themes of a random selection of 207 English tweets in the 6 p.m. sample are summarized in Table 3.

Table 3. Content of a random sample of English tweets

\begin{tabular}{|c|c|c|c|}
\hline & w/@ & w/o@ & Total \\
\hline $\begin{array}{l}\text { about } \\
\text { addressee }\end{array}$ & $\begin{array}{c}21 \\
(33 \%)\end{array}$ & $\begin{array}{c}2 \\
(1 \%)\end{array}$ & $\begin{array}{c}23 \\
(11 \%)\end{array}$ \\
\hline $\begin{array}{l}\text { announce/ } \\
\text { advertise }\end{array}$ & $\begin{array}{c}0 \\
(0 \%)\end{array}$ & $\begin{array}{c}14 \\
(10 \%)\end{array}$ & $\begin{array}{c}14 \\
(7 \%)\end{array}$ \\
\hline exhort & $\begin{array}{c}7 \\
(11 \%)\end{array}$ & $\begin{array}{c}1 \\
(1 \%)\end{array}$ & $\begin{array}{c}8 \\
(4 \%)\end{array}$ \\
\hline $\begin{array}{l}\text { info for } \\
\text { others }\end{array}$ & $\begin{array}{c}10 \\
(16 \%) \\
\end{array}$ & $\begin{array}{c}1 \\
(1 \%) \\
\end{array}$ & $\begin{array}{c}11 \\
(5 \%) \\
\end{array}$ \\
\hline $\begin{array}{l}\text { info for } \\
\text { self }\end{array}$ & $\begin{array}{c}2 \\
(3 \%)\end{array}$ & $\begin{array}{c}9 \\
(6 \%) \\
\end{array}$ & $\begin{array}{c}11 \\
(5 \%) \\
\end{array}$ \\
\hline $\begin{array}{l}\text { meta-com- } \\
\text { mentary }\end{array}$ & $\begin{array}{c}0 \\
(0) \\
\end{array}$ & $\begin{array}{c}4 \\
(3 \%) \\
\end{array}$ & $\begin{array}{c}4 \\
(2 \%) \\
\end{array}$ \\
\hline $\begin{array}{l}\text { media } \\
\text { use }\end{array}$ & $\begin{array}{c}4 \\
(6 \%) \\
\end{array}$ & $\begin{array}{c}14 \\
(10 \%) \\
\end{array}$ & $\begin{array}{c}18 \\
(9 \%) \\
\end{array}$ \\
\hline $\begin{array}{l}\text { express } \\
\text { opinion }\end{array}$ & $\begin{array}{c}5 \\
(8 \%) \\
\end{array}$ & $\begin{array}{c}8 \\
(6 \%)\end{array}$ & $\begin{array}{c}13 \\
(6 \%) \\
\end{array}$ \\
\hline $\begin{array}{l}\text { other's } \\
\text { experience }\end{array}$ & $\begin{array}{c}1 \\
(2 \%)\end{array}$ & $\begin{array}{c}10 \\
(7 \%)\end{array}$ & $\begin{array}{c}11 \\
(5 \%)\end{array}$ \\
\hline $\begin{array}{l}\text { self } \\
\text { experience }\end{array}$ & $\begin{array}{c}11 \\
(17 \%) \\
\end{array}$ & $\begin{array}{c}73 \\
(51 \%) \\
\end{array}$ & $\begin{array}{c}84 \\
(41 \%) \\
\end{array}$ \\
\hline $\begin{array}{l}\text { solicit } \\
\text { info }\end{array}$ & $\begin{array}{c}0 \\
(0) \\
\end{array}$ & $\begin{array}{c}3 \\
(2 \%)\end{array}$ & $\begin{array}{c}3 \\
(1 \%) \\
\end{array}$ \\
\hline $\begin{array}{l}\text { other } \\
\text { misc }\end{array}$ & $\begin{array}{c}2 \\
(3 \%) \\
\end{array}$ & $\begin{array}{c}5 \\
(3 \%) \\
\end{array}$ & $\begin{array}{c}7 \\
(3 \%) \\
\end{array}$ \\
\hline Total & 63 & 144 & 207 \\
\hline
\end{tabular}

The most popular content of tweets is reporting one's own experience, consistent with the stated purpose of Twitter to answer the question "What are you doing?" Fifty-one percent of tweets without @ signs, and 17\% of tweetswith@ signs, appear to address this question.

Moreover, interesting differences can be observed between the content of tweets with and without the @ sign. Tweets with@ signs are more focused on an addressee, more likely to provide information for others, and more likely to exhort others to do something - in short, their content is more interactive. In contrast, tweets without @ signs are more self-focused, although they also report other's experiences, and they make more general announcements. The low incidence of addressee orientation $(2 \%)$ and exhortations $(1 \%)$ in tweets without@ @uggests that, while users may choose in theory to respond to other tweets without using the@ sign, in practice such unmarked responses are infrequent.

\subsection{Responses to@}

Because an initiation must receive a response in order for conversation to occur, the fourth research question asked how many messages directed to others received one or more responses. To answer this question, we examined all English messages with and without @ signs posted to Twitter within the first 30 minutes of the 6 p.m. sample and identified public responses (by searching for the @ sign) appearing in the remainder of the hour. Messages without @ signs were included in this analysis, because we observed that some undirected messages (without @ signs) were responded to, and thus became post facto initiations. However, it was less common for responders not to use an @ sign to indicate the addressee, as noted above.

This is a very conservative measure of responsiveness, in that it does not include responses that were posted after the one-hour time frame, responses that did not employ the@ sign, or private responses. Even without including these, of the 785 public English tweets that used the @ sign to direct the message to a particular individual that were posted in that half hour, 245 messages $(31.2 \%)$ received a public response.

This rate is considerably higher than the rate of $7 \%$ reported by Zelenkauskaite and Herring [18] for public responses received to iTV SMS within a two-day period. Moreover, the actual response rate to tweets with (a) signs, including data not accessible to us, is almost certainly higher. This measure thus suggests that the responsiveness of Twitter as a conversational environment is at least at the low end of moderate and probably higher. 


\subsection{Coherence of Twitter conversations}

The fifth research question asked: How long, and how coherent, are Twitter conversations? The 6 p.m. sample contained 123 exchanges in English, where an exchange was defined as at least one initiation and one response involving at least two people, with or without use of the @ sign. Table 4 shows descriptive statistics of the exchanges for five categories: the number of participants in each exchange; the length of the exchange (within the one-hour time frame); the number of exchanges; the percentage of tweets employing the (a) sign to address the tweet to a participant; and the average time between tweets within the exchange.

Table 4. Characteristics of Twitter conversations

\begin{tabular}{|l|c|c|c|c|}
\hline & Range & Mean & Median & Mode \\
\hline \# Participants & $2-10$ & 2.5 & 2 & 2 \\
\hline $\begin{array}{l}\text { Length } \\
\text { (min:sec) }\end{array}$ & $\begin{array}{c}00: 25- \\
54: 22\end{array}$ & $17: 41$ & $26: 33$ & - \\
\hline \# Exchanges & $2-30$ & 4.62 & 3 & 3 \\
\hline $\begin{array}{l}\text { \% Tweets } \\
\text { using @ per } \\
\text { exchange }\end{array}$ & $\begin{array}{c}20 \%- \\
100 \%\end{array}$ & $86 \%$ & $100 \%$ & $100 \%$ \\
\hline $\begin{array}{l}\text { Avg time bt } \\
\text { tweets in ex- } \\
\text { changes }\end{array}$ & $\begin{array}{c}00: 25- \\
34: 05\end{array}$ & $06: 43$ & $04: 24$ & - \\
\hline
\end{tabular}

To the extent that this sample is representative, most conversations that occur in Twitter appear to be dyadic exchanges of three to five messages sent over a period of 15 to 30 minutes. The rate of using the @ sign to address a message to another participant in these conversations is very high, with a mean of $86 \%$ and a median and mode of $100 \%$. This further supports the view that the @ sign is closely associated with conversational activity.

To examine the coherence of these exchanges, two conversations - one involving a small number of participants (mostly two people, with two others contributing one message each) and another with 10 participants-were charted using VisualDTA. In addition to illustrating a mostly dyadic exchange and a longer, more complex group exchange, these conversations were selected because both involve collaborative information sharing.

In the first exchange, which is 20 messages long and lasts just over 41 minutes, camh twitters about a problem she or he is having with some Twitter settings. Elliotcable and two other Twitter users respond with advice and questions intended to diagnose camh's problem. camh directs them to a URL with a screenshot illustrating the problem settings, and after some discussion, the conversation drifts towards commenting on the tool used to create the screen capture.

Figure 2 displays the evolution of topics in this exchange over time as diagramed using VisualDTA. In DTA diagrams, $T$ indicates a proposition that is narrowly on-topic with the proposition to which it responds, while P indicates a parallel shift that introduces a new idea into the discourse. The y-axis represents the messages in chronological order, and the $\mathrm{x}$-axis represents cumulative semantic distance between propositions. Figure 2 shows that this conversation is continuous and proceeds in a mostly gradual, step-wise fashion (via parallel shifts with a semantic distance of 1) from the upper left to the lower right. This pattern, including its tendency toward topic drift, is characteristic of a coherent dyadic conversation, such as takes place, for example, via instant messaging [18].

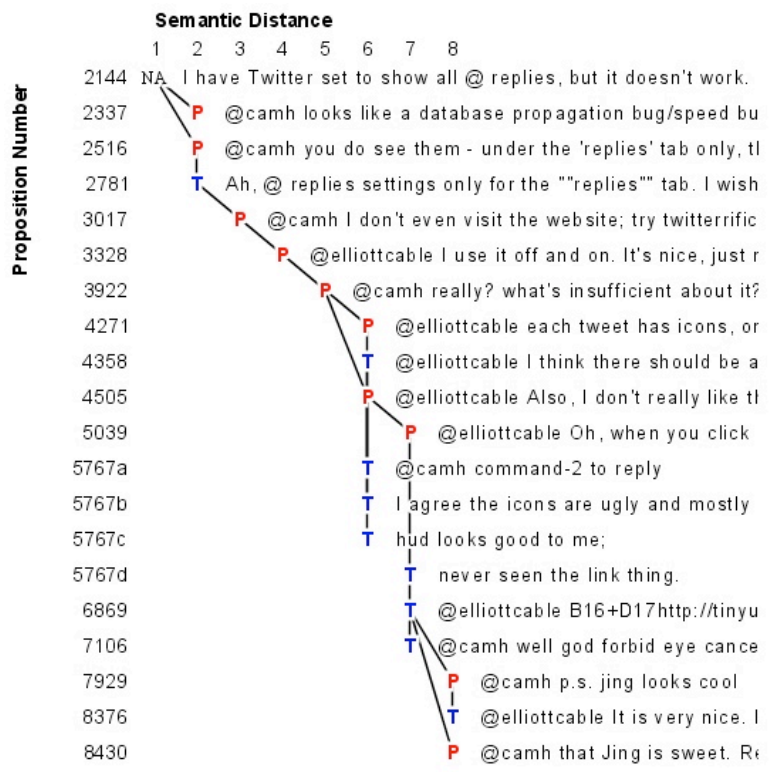

Figure 2. VisualDTA diagram of a mostly-dyadic conversation

The second conversation is 32 messages long and lasted 31 minutes. It was initiated by courosa, who publicly announces (in three undirected tweets) his or her discovery of a website for a fraudulent academic conference. Others respond quickly (using @ signs) with reactions, further observations about the site based on their own examination, suggestions regarding how to respond to it (including the URL of a phishing site where it can be reported), and suggestions in response to courosa's request for a "good site saver."

The topical evolution of this group conversation is complex, as shown in Figure 3. Because the responses to courosa's initial tweets came quickly, many messages overlapped each other temporally, with the result 
that several simultaneous sub-threads developed. Moreover, while some gradual, diagonal drift is evident, the overall shape of the VisualDTA diagram resembles a set of reactions to an initial prompt more than a collaboratively negotiated, gradually developing conversation. However, even though it is less coherent than the conversation in Figure 2, this group conversation still accomplishes the exchange of information and the collaborative development of ideas.

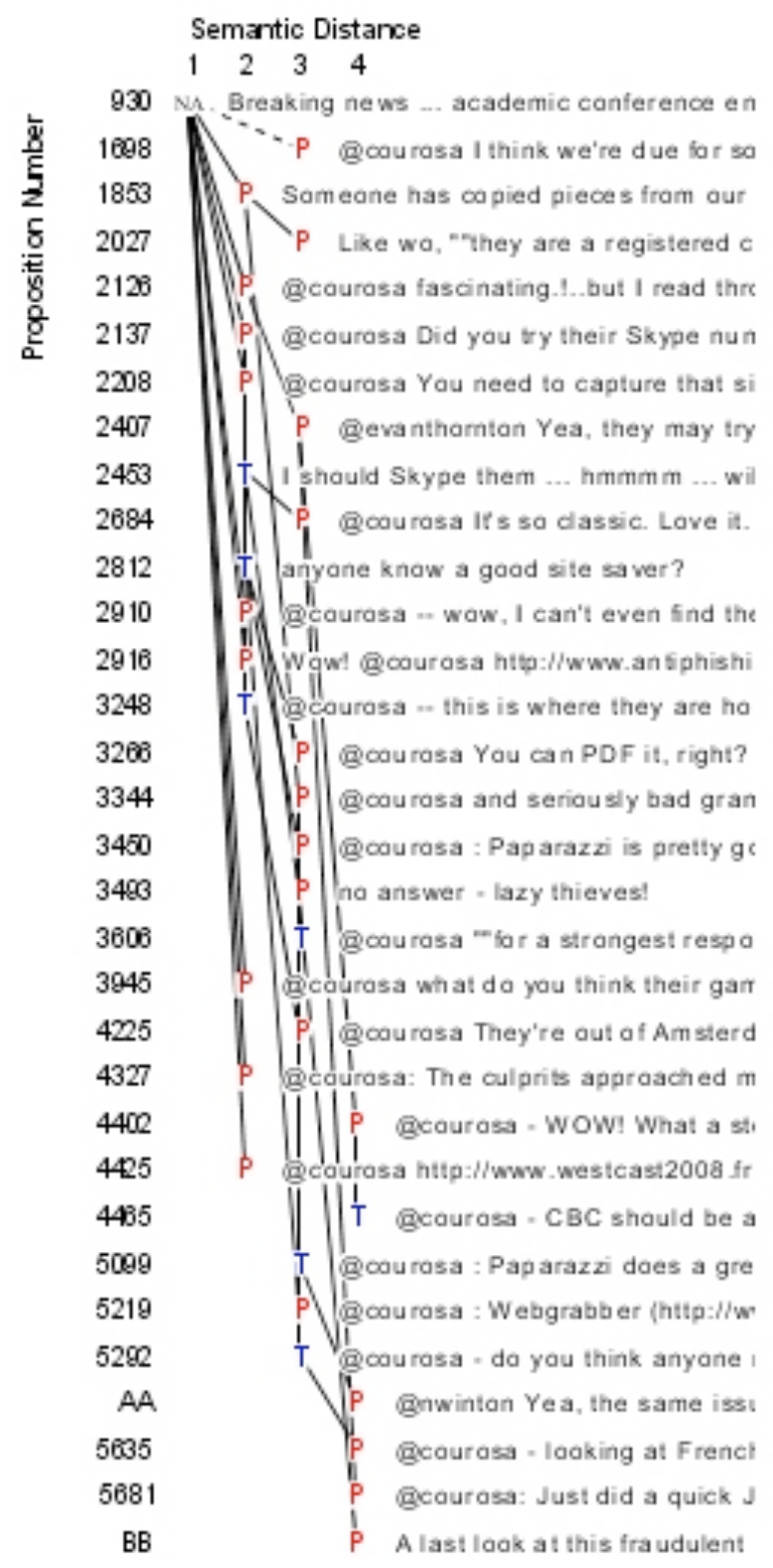

Figure 3. VisualDTA diagram of a conversation with
10 participants

The mostly-dyadic conversation in Figure 2 is typical of the Twitter conversations in our sample in terms of its coherence, although it is somewhat longer than average. The multi-participant conversation in Figure 3 is atypical; it is the longest, most complex interaction in our sample. However, it illustrates the level of interactional complexity that is possible in Twitter. It also reveals some of Twitter's limitations as a tool for conversation and collaboration.

\section{Discussion}

This study investigated the conversationality of Twitter, with special attention to the role played by the (a) sign. Our first research question asked about the breakdown of the language of tweets across time periods, and how often the @ sign is used in tweets across languages. English speakers were found to be the most dominant language group present in our sample, which is not surprising, given that Twitter originated in the U.S. However, Japanese and Spanish speakers were also well represented in our corpus. The @ sign was used at roughly the same rate (around 30\%) in all the languages for which enough tweets were available to analyze, more than double the rate of @ usage reported only two years ago [8]. Thus it seems that use of Twitter as a tool for interpersonal interaction is spreading globally.

The second research question asked: How do instances of the@ sign function in English tweets? We found support in our data for the assumption in previous Twitter studies that the presence of the @ sign signifies that the tweet is part of a conversation. More than 90\% of tweets with@ addressed an individual, as opposed to having some other function (although a range of other functions was also noted).

Moreover, the most interactive contentexhortations and content relating to an addressee-was overwhelmingly found in tweets with @ signs, as shown in Table 3. This partially addresses the third research question, which asked if there were content differences between tweets with and without the@ sign. We further found that tweets with@ exhibited a wider range of content, in comparison to tweets without@, and that most tweets without@just answered the Twitter site's question: What are you doing? This suggests that@, in addition to directly enabling a more interactive use of Twitter, is indirectly contributing to expanding the types of content expressed in tweets. ${ }^{2}$

In response to the fourth research question-to what extent do English@ messages that are directed to others receive responses?-a conservative measure of

\footnotetext{
${ }^{2}$ We also have observed that the addressive use of @ is spreading to other contexts, such as Flickr comments, in which the interface does not provide users a mechanism for indicating that a message is responding to another message.
} 
public responses posted within a one-hour period indicated that about 31\% of tweets with@ received a response. On the one hand, this suggests that some (perhaps large) number of initiations falls by the wayside in Twitter, which could be a problem for task-related communication, in which initiations usually require a response. On the other hand, without knowing how many responses were sent privately, we recommend interpreting this result with caution. Viewed in a more positive light, it indicates that even in the current noisy Twitter environment, successful exchanges can and do take place.

The latter view is further supported by the results of the analysis of Twitter conversations. A one-hour sample was found to include an average of about two English-language exchanges per minute, typically short and typically taking place between two users. At the same time, longer, multi-participant interactions were also identified. The VisualDTA diagrams showed that topical development in the service of collaborative problem solving can take place in Twitter and that conversations can progress quite coherently. However, the larger the number of participants, the greater the risk that threads will cross and topical coherence will suffer. This is true for other modes of text-only computer-mediated communication as well, of course [5]. In the context of this study, it suggests that if Twitter is used for collaboration, communication in dyads or small groups would be more effective than large, open discussions.

\section{Conclusions}

Some people might argue that Twitter was not designed for collaboration, and therefore should not be critiqued in conversational terms. However, the examples of extended conversations presented here show that some users are already taking advantage of Twitter for informal collaborative purposes, and conversation is an essential component of collaboration. We predict that tools such as Twitter will soon come to be used in formal collaborative contexts, as well-for example, in work involving distributed teams, much like instant messaging before them [13]. "Microblogging" has the potential to add lightweight, mobile access to a repertoire of older CMC tools that are bound to a computer. Its provision of a web repository also fits well with the ongoing trend to store digital documents, including CMC archives, online.

The current Twitter interface presents a number of challenges that serious users must overcome, however. These include: a limited number of tweets the user is able to receive per refresh and automatic refresh intervals of not less than one minute, which cause responses potentially to be missed; a limited history of tweets from followers, which is not preserved; lack of an interface view that displays tweets directed to and received from the same users (i.e., all parts of an exchange in one place); and the absence of a search function. At present, the use of third party clients, individual Twitter homepages, and-of course-the@ sign help create coherence in the Twitter environment.

Design modifications could make microblogging platforms such as Twitter more suitable for collaboration, and may be advisable if such platforms become widely adopted. For example, more persistent archives of the tweets of "followers," as well as interface views that allow all contributions to an exchange to be viewed together on a user's homepage, would increase the usability of Twitter for conversational purposes. Customizable individual and group spaces would also provide more controlled environments in which to meet and communicate with collaborators.

There is also the problem of the sheer size of the Twitter universe and the difficulty of tracking tweets from someone whom one may not already be "following." According to [4], the Twitter developers acknowledge the need for a search function, and initially had it in the design but had to take it out due to "scaling issues." 3 The developers suggest that the fact that one has to hunt oneself for someone can make a large network seem more closed and personal. However, while this may be true for recreational communication environments (something similar is suggested for iTV SMS in [18]), collaborative teams and task-focused groups, whether in work or educational environments, generally prefer to minimize unnecessary effort.

This study is limited by the fact that only the 12hour period from 6 a.m. to 6 p.m. EST was sampled. This could have created a bias toward users in the western hemisphere. Future studies should collect data at regular intervals over a 24-hour period to arrive at a more complete picture of Twitter usage in different time zones. It would also be valuable to repeat this analysis at a later point to assess the rate at which different language groups increase or decrease their use of Twitter. Additionally, a Japanese-language version of Twitter was released in April 2008, and Twitter-like tools have been developed for use in other cultures [17]; these also deserve study.

Finally, a precondition for successful adoption of a new technology tool (or a new use of an existing tool) is a positive attitude towards its potential. Analyses of user-created content such as were conducted in this study should be supplemented with ethnographic studies and interviews with Twitter and non-Twitter users.

\footnotetext{
${ }^{3}$ In July 2008, Twitter acquired a startup called Summarize that had created a function allowing users to search Twitter messages; it is available at the sub-domain search.twitter.com [17].
} 
A further study might seek to identify the $2 \%$ of users in Odden's [10] study who reported using Twitter for "group and project communication" and seek to understand their experiences, good and bad. More generally, serious user perspectives, and not just those of users for whom tools such as Twitter are an interesting novelty, should inform future research and development of microblogging systems, as microblogging evolves to become more conversational and collaborative.

\section{References}

[1] Bays, H. (1998). Framing and face in Internet exchanges: A socio-cognitive approach. Linguistik Online, 1(1). Retrieved May 20, 2008 from http://www.linguistikonline.de/bays.htm

[2] Diaz, S. (2007, June 9). Life, in little chirps: Introducing Twitter, a web experience in the mass appeal of mundane details. The Washington Post. Retrieved May 21, 2008 from http://www.washingtonpost.com/wp-dyn/content/article/2007 /06/08/AR2007060802614.html

[3] Freiert, M. (2008, May 15). Twitter traffic explosion: Who's behind it all? Compete. Retrieved May 21, 2008 from http://blog.compete.com/2008/05/15/twitter-traffic-growthusage-demographics/

[4] Glaser, M. (2007, May 17). Twitter founders thrive on micro-blogging constraints. Media Shift. Retrieved May 21, 2008 from http://www.pbs.org/mediashift/2007/05/ digging_deepertwitter_founders.html

[5] Herring, S. C. (1999). Interactional coherence in CMC. Journal of Computer-Mediated Communication, 4(4). Retrieved May 27, 2008 from http://jcmc.indiana.edu/vol4/ issue $4 /$ herring.html

[6] Herring, S. C. (2003). Dynamic topic analysis of synchronous chat. In: New Research for New Media: Innovative Research Methodologies Symposium Working Papers and Readings. Minneapolis, MN: University of Minnesota School of Journalism and Mass Communication. Retrieved June 15, 2008 from http://ella.slis.indiana.edu/ herring/dta.2003.pdf

[7] Herring, S. C., \& Kurtz, A. J. (2006). Visualizing Dynamic Topic Analysis. Proceedings of CHI'06. ACM Press: New York. Retrieved September 8, 2008 from http://ella.slis.indiana.edu/ herring/chi06.pdf

[8] Java, A., Song, X., Finn, T., \& Tseng, B. (2006, August). Why we Twitter: Understanding microblogging usage and communities. Joint 9th WEBKDD and 1st SNA-KDD Workshop '07, San Jose, CA.

[9] Mischaud, E. (2007). Twitter: Expressions of the whole self. An investigation into user appropriation of a web-based communications platform. London: Media@1se. Retrieved May 20, 2008 from http://www.lse.ac.uk/collections/ media@1se/mediaWorkingPapers/MScDissertationSeries/Misch aud_final.pdf

[10] Odden, L. (2008, March 5). Reader poll: How do you use Twitter? Online Marketing Blog. Retrieved May 22, 2008 from http://www.toprankblog.com/2008/03/reader-poll-howdo-you-use-twitter/

[11] Panyametheekul, S., \& Herring, S. C. (2007). Gender and turn allocation in a Thai chat room. In B. Danet \& S. C. Herring (Eds.), The Multilingual Internet: Language, Culture, and Communication Online (pp. 233-255). New York: Oxford University Press.

[12] Pontin, J. (2007, April 22). From many tweets, one loud voice on the internet. The New York Times. Retrieved May 21, 2008 from http://www.nytimes.com/2007/04/22/business/yourmoney/22stream.html?partner $=$ rssnyt\&emc $=$ rss

[13] Quan-Haase, A., Cothrel, J., \& Wellman, B. (2005). Instant messaging for collaboration: A case study of a hightech firm. Journal of Computer-Mediated Communication, 10(4), article 13. Retrieved June 12, 2008 from http://jcmc.indiana.edu/vol10/issue4/quan-haase.html

[14] Sacks, H. E., Schegloff, E., \& Jefferson, G. (1974). A simplest systematics for the organization of turn-taking for conversation. Language, 50, 696-735.

[15] Twitter.com. (2008). Twitter home page. Retrieved May 21, 2008 from http://twitter.com/home

[16] Werry, C. C. (1996). Linguistic and interactional features of Internet Relay Chat. In S. C. Herring (Ed.), Computer-mediated communication: Linguistic, social and crosscultural perspectives (pp. 47-63). Amsterdam: John Benjamins.

[17] Wikipedia. (2008, September 7). Twitter. Retrieved September 7, 2008 from http://en.wikipedia.org/wiki/Twitter.

[18] Zelenkauskaite, A., \& Herring, S. C. (2008). Televisionmediated conversation: Coherence in Italian iTV SMS chat. Proceedings of the Forty-First Hawai'i International Conference on System Sciences (HICSS-41). Los Alamitos, CA: IEEE Press. 